\title{
Insights from inside the spinodal: Bridging thermalization time scales with smoothed particle hydrodynamics
}

\author{
Martin Pütz and Peter Nielaba \\ Universität Konstanz, Fachbereich für Physik, 78457 Konstanz, Germany
}

(Received 22 April 2016; published 30 August 2016)

\begin{abstract}
We report the influence of the strength of heat bath coupling on the demixing behavior in spinodal decomposing one component liquid-vapor systems. The smoothed particle hydrodynamics (SPH) method with a van der Waals equation of state is used for the simulation. A thermostat for SPH is introduced that is based on the Berendsen thermostat. It controls the strength of heat bath coupling and allows for quenches with exponential temperature decay at a certain thermalization time scale. The present method allows us to bridge several orders of magnitude in the thermalization time scale. The early stage is highly affected by the choice of time scale. A transition from exponential growth to a $1 / 2$ ordinary power law scaling in the characteristic lengths is observed. At high initial temperatures the growth is logarithmic. The comparison with pure thermal simulations reveals latent heat to raise the mean system temperature. Large thermalization time scales and thermal conductivity are figured out to affect a stagnation of heating, which is explained with convective processes. Furthermore, large thermalization time scales are responsible for a stagnation of growth of domains, which is temporally embedded between early and late stage of phase separation. Therefore, it is considered as an intermediate stage. We present an aspect concerning this stage, namely that choosing larger thermalization time scales increases the duration. Moreover, it is observed that diffuse interfaces are formed during this stage, provided that the stage is apparent. We show that the differences in the evolution between pure thermal simulations and simulations with an instantaneously scaled mean temperature can be explained by the thermalization process, since a variation of the time scale allows for the bridging between these cases of limit.
\end{abstract}

DOI: 10.1103/PhysRevE.94.022616

\section{INTRODUCTION}

The coexistence of separated phases, especially between liquid and vapor, but also separation of liquid mixtures or foam formation and growth, are fundamental dynamic processes in our everyday life. However, it is not yet fully clarified which physical processes are totally involved to it. The understanding of the dynamics of phase separation contributes to the understanding of even more complicated systems, such as in colloidal suspensions or polymer solutions. Still open issues concern the role of hydrodynamics and the thermal evolution of the phases from the very beginning on, when the fluid is still in the initial state and not separated. For the purpose of demixing, an initially stable system is quenched to an unstable state below the coexistence region. The quench is typically driven by a temperature change via coupling to an external heat bath, but also volume quenches are technically feasible. The depth of the quench defines the responding kind of demixing. Close beneath the coexistence region the state becomes metastable, which is the case for superheated liquids or supercooled vapor, and the separation occurs as a rare event induced by statistical superposition of fluctuations or by adding an external nucleus. As the quench deepens the system becomes completely unstable and a special type of phase separation takes place. It is called spinodal decomposition, wherein the system isotropically initiates to separate throughout the whole volume. A detailed review can be found in Ref. [1].

Once separated, the type of coupling to an external heat bath, computationally realized by so called thermostats [2], is also a decisive factor in the further evolution of the system. Thus, a very strong coupling results in isothermal systems [3] or, at least, quasi-isothermal with thermal fluctuations [4].
However, there is strong evidence for nonisothermal behavior [5,6]. Contrarily, very weak coupling allows for a pure thermal treatment of the system, consequentially followed by the latent heating of the system [5]. The comparison between experimental practice [7,8] and numerical [5], but also theoretical concepts [9], reveals a discrepancy. The limits of both pure thermal and instantaneous scaling thermostats are hardly realizable. Therefore, the most natural and closest to experiment approach is to model a thermostat that allows for time dependent temperature relaxation at a certain rate.

One approach for the theoretical description is to implement all physical principles that are relevant to the dynamics of phase separation. The demixing behavior of the system naturally arises from the principles, such as the cohesive pressure and covolume in the van der Waals (vdW) theory [10] for liquid-vapor systems. These basic ideas of vdW are further extended to binary mixtures, culminating in the phenomenological Cahn-Hilliard $(\mathrm{CH})$ equation $[9,11]$. The $\mathrm{CH}$ approach excellently reproduces the behavior of isothermal systems, but it does not include hydrodynamic interactions [12]. However, our approach is to describe the fluid flow with the hydrodynamic equations, which are given by a set of partial differential equations. The set is closed by the vdW equation of state (EOS) $[13,14]$. This vdW approach naturally provides surface tension and can intrinsically form diffuse interfaces [6].

The dynamics of spinodal decomposition splits up in an early and a late stage, even though they are not clearly separated, and depends on the actual choice of system parameters.

In the early stage spontaneous demixing occurs. The so called "homophase fluctuations" arise and depend on initial noise of density fluctuations. This growth of fluctuations 
happens very fast and is, therefore, hardly observable in experiments. A discussion about the growth and the importance of finite quench rates for realistic models can be found in Ref. [1]. However, in the further evolution a stagnation in the growth can occur, which is interpreted as an intermediate stage [3,5,15-17]. The late stage manifests where the actually separated domains begin to grow. The growth of the characteristic length scale of the system can generally be described by ordinary power law scaling. Various former theoretical studies focus on predictions for the coarsening process in this late stage and the driving physical processes [18-20], whereafter the actual growth rates are given by the diffusion driven Lifshitz-Slyozov (LS) growth [21] and the viscous (VH) and inertial hydrodynamics (IH) growth regime [22]. A crossover between regimes has been found [23]. Moreover, there is also evidence for a connection between the actual local temperature and the time of initialization of the hydrodynamic regime, such that the regimes can even overlay each other [6]. Since many investigations and comparisons exist concerning this late stage and has become a well studied phenomenon, only few exist for the early stage. Therefore, the main focus of our work will be on the dynamics of the latter.

We use the smoothed particle hydrodynamics (SPH) method for our simulations, which has several advantages in the context of phase separation compared to other simulation techniques. Thus, it allows us to directly follow the path of the separated phases through the phase diagram. Furthermore, the characteristic lengths of the domains are easily calculated without difficult calculations via structure factor and Fourier transforms [6]. Our simulation method expands the fund of methods for realistic treatment of spinodal decomposition and provides deeper insights to the physics involved in phase separation processes.

\section{METHOD}

For this work a modification of Gadget-2 [24], a massively parallelized tree based smoothed particle hydrodynamics (SPH) code, is used, originally written for astrophysical purposes. The modifications comprise a smoothing kernel, an equation of state that provides phase separation, a thermal conduction equation, and an SPH thermostat. Here, only a brief summary of the governing equations and modifications of the basic SPH algorithm is given. More detailed descriptions of the concepts of SPH can be found in the latest reviews by Springel [25], Price [26], and Monaghan [27].

\section{A. Smoothed particle hydrodynamics}

The SPH method is a Lagrangian based meshless particle method, which means, that the interpolation grid is not fixed, but given by comoving so called SPH particles, where every particle is the origin of its own comoving reference frame. The objective of SPH is to solve the hydrodynamic equations, a set of partial differential equations. In Lagrangian formulation the first is the continuity equation, which is described by

$$
\frac{d \rho}{d t}=-\rho \nabla \cdot \mathbf{v}
$$

where $\rho$ is the mass density, $\mathbf{v}$ is the velocity, and $d / d t=$ $\partial / \partial t+(\mathbf{v} \cdot \nabla)$ denotes the convective derivative. Depending on whether viscosity is taken into account or not, the momentum equation is called the Navier-Stokes equation, or Euler equation, respectively, and is written as

$$
\frac{d \mathbf{v}}{d t}=-\frac{\nabla P}{\rho},
$$

where $P$ is the pressure. For the Navier-Stokes equation, $P$ can be understood as the stress tensor. It additionally has viscous stress terms included. In contrast, in the case of the Euler equation, $P$ has only diagonal pressure entries. The third equation corresponds to the first law of thermodynamics, $d U=\delta Q+\delta W$, where $\delta Q$ expresses the thermal exchange with an external heat bath and $\delta W$ is the work done in the system, the energy equation. In hydrodynamics, it is mostly described in terms of a specific internal energy $u$,

$$
\frac{d u}{d t}=-\frac{P}{\rho} \nabla \cdot \mathbf{v}-\frac{1}{\rho} \nabla \mathbf{j},
$$

where $\mathbf{j}$ is the heat flux vector. Note that the traditional formulation of SPH is adiabatic, and therefore there is no heat exchange $\delta Q=0$, although it is basically not obligatory and will be used in this work for the implementation of a thermostat. Thermal exchange within the simulation volume is given by the heat flux vector $\mathbf{j}=\kappa \nabla T$, where $T$ is the local temperature and the $\kappa$ is the thermal conductivity.

Following the main idea of SPH, the simulation volume is subdivided into a set of elements of a given constant mass $m$, the SPH particles. Every SPH particle is spatially extended, where a smoothing length $h$ determines the width of the smoothing profile, which is based on a Gaussian shaped kernel function. In practice, the Gaussian distribution itself is not the first choice, whereas normalized polynomials with compact support are commonly used to enhance the computational efficiency. Here, the Wendland $C_{4}$ kernel

$$
W(\mathbf{r}, h)=\frac{495}{32 \pi}(1-r)_{+}^{6}\left(1+6 r+\frac{35}{3} r^{2}\right)
$$

is used, where $h$ is the smoothing length, $r=|\mathbf{r}| / h$ and $(\cdot)_{+}=\max (0, \cdot)[28]$. Thus the support of the kernel depends only on $h$. The class of Wendland kernels has been shown to yield excellent results and has outstanding features compared to other common choices [29,30]. The smoothed particles are penetrating each other and affect the behavior of their neighboring particles, within the smoothing range. Instead of integrating Eq. (1), the density is estimated by a summation over all SPH particles, where the masses contribute to the density with a weighting given by the kernel, thus, in practice, only the neighboring particles need to be considered. The estimation for a particle $i$ reads as

$$
\rho_{i}=\sum_{j=1}^{N} m_{j} W_{i j}\left(h_{i}\right)
$$

where $W_{i j}\left(h_{i}\right)=W\left(\mathbf{r}_{i}-\mathbf{r}_{j}, h_{i}\right)$. One of the main advantages of the method can be seen from Eq. (5). Since the number of particles in the simulation volume is constant and the kernel is normalized, the total mass of the system is conserved [26]. The smoothing length $h_{i}$ is usually adaptively chosen and determined iteratively, such that the volume, defined by $h_{i}$, encloses a constant mass [24], where the number of neighbors 
$N$ has turned out to be a suitable initial parameter for this calculation. Note that any physical quantity can be expressed in an analogous way. The SPH expression of the spatial derivatives from Eqs. (2) and (3), but also for any other physical quantity, are also reduced to a sum, where the derivatives only operate on the kernel function, since the quantity is only scalar and the field properties are reduced to the kernel, of which the derivative can easily be calculated analytically. In the symmetrized formulation with adaptive smoothing lengths, Eq. (2) reads as

$$
\frac{d \mathbf{v}_{i}}{d t}=-\sum_{j}^{N} m_{j}\left[\frac{f_{i} P_{i}}{\rho_{i}^{2}} \nabla_{i} W_{i j}\left(h_{i}\right)+\frac{f_{j} P_{j}}{\rho_{j}^{2}} \nabla_{i} W_{i j}\left(h_{j}\right)\right],
$$

where the $f_{i}$ are correction factors, defined by

$$
f_{i}=\left[1+\frac{h_{i}}{3 \rho_{i}} \frac{d \rho_{i}}{d h_{i}}\right]^{-1} .
$$

The SPH expression for the change of internal energy in Eq. (3) is subdivided into the work term,

$$
\frac{d u_{i}}{d t}=\frac{f_{i} P_{i}}{\rho_{i}^{2}} \sum_{j}^{N} m_{j}\left(\mathbf{v}_{i}-\mathbf{v}_{j}\right) \cdot \nabla_{i} W_{i j}\left(h_{i}\right),
$$

and the heat term, which is the spatial derivative of heat flux and, therefore, a second derivative of the temperature $[6,31,32]$. It is given by

$$
\frac{d u_{i}}{d t}=\sum_{j=1}^{N} \frac{m_{j}}{\rho_{i} \rho_{j}}\left(\kappa_{i}+\kappa_{j}\right)\left(T_{j}-T_{i}\right) \frac{\mathbf{r}_{i j}}{\left|\mathbf{r}_{i j}\right|^{2}} \cdot \nabla_{i} \bar{W}_{i j},
$$

where $\bar{W}_{i j}=1 / 2\left[W_{i j}\left(h_{i}\right)+W_{i j}\left(h_{j}\right)\right]$ is the arithmetic mean between the two kernel values. The thermal conductivity $\kappa$ is modeled by a density dependency

$$
\kappa_{i}=\kappa_{0} \rho_{i},
$$

where $\kappa_{0}$ is a constant parameter [33]. The SPH method is known to produce unphysical behavior in the presence of shocks. In order to improve the method, the standard implementation of artificial viscosity in Gadget-2 is used [24]. The main idea is to suppress unphysical behavior in shocks, that arises when SPH particles approach each other. Artificial viscosity acts like real physical viscosity by an interparticle term

$$
\Pi_{i j}=\frac{\alpha v_{i j}^{\text {sig }} w_{i j}}{2 \bar{\rho}_{i j}},
$$

which is added to the pressure in Eqs. (6) and (8), with the artificial viscosity constant $\alpha=1$ and a signal velocity is defined by $v_{i j}^{\text {sig }}=c_{i}+c_{j}-3 w_{i j}$ with $w_{i j}=\mathbf{v}_{i j} \cdot \mathbf{r}_{i j} /\left|\mathbf{r}_{i j}\right|$ (but only if $\mathbf{v}_{i j} \cdot \mathbf{r}_{i j}<0$, otherwise $w_{i j}=0$ ). The $c_{i}$ is an estimate of the sound velocity and $\bar{\rho}_{i j}$ is the arithmetic mean of $\rho_{i}$ and $\rho_{j}$. Note that artificial viscosity is only improving the numerical stability and must not be confused with real physical viscosity.

The set of hydrodynamic equations are closed by an equation of state (EOS). In the context of phase separation of one-component fluids, it is appropriate to follow the van der Waals (vdW) approach [10,13,14], which is given by two
EOSs, namely the mechanic

$$
P_{i}=\frac{\rho_{i} \bar{k}_{b} T_{i}}{1-\bar{b} \rho_{i}}-\bar{a} \rho_{i}^{2},
$$

and the caloric equation

$$
u_{i}=\bar{k}_{b} T_{i}-\bar{a} \rho_{i}
$$

where $\bar{k}_{b}=1, \bar{a}=2.0$, and $\bar{b}=0.5$ are the reduced units of the Boltzmann constant, the cohesive pressure, and the covolume, respectively. The $\mathrm{vdW}$ theory predicts a certain unstable region, of which a system, quenched into this region, initiates to separate in liquid and vapor phases, where thermal equilibrium is reached on the so called binodal curve of phase coexistence. A quenched fluid becomes more and more inseparable with increasing temperature culminating in the critical point $\left(P_{c}, T_{c}, \rho_{c}\right)$, where the state of the phase becomes undefinable. The covolume and cohesive pressure can also be expressed in terms of the critical point; see, e.g., Ref. [6]. The vdW EOSs have already been applied to SPH for droplet formation, deformation, and coalescence [34-38]. All former studies have in common that appropriate results were only produced if the force calculation was split in long and short ranging components, with unequal smoothing lengths. From the SPH point of view, there is no explanation for this choice. It is also one of the advantages that must be addressed to the Wendland kernels, that produce excellent results without the splitting procedure [6].

The time integration is done by the kick-drift-kick algorithm. An adaptive time-stepping leapfrog scheme with excellent conservation properties is used, where the time steps are chosen individually, based on a binary hierarchy [24], bounded by a maximum step size $\Delta t_{\max }$. A detailed description of the time-step conditions, used herein, can be found in Ref. [6]

\section{B. Thermalization by heat bath coupling}

As mentioned in Sec. II A, it is not necessary to treat the simulations as adiabatic, where $\delta Q=0$, but to model thermal exchange with an external heat source. These so called thermostats are common methods to treat temperature realistic in microscopic simulations, such as molecular dynamics (MD) or Monte Carlo [2]. The transfer of thermostats from MD to SPH is obvious, since the methods are very similar [39] and a simple scaling thermostat has already been applied to SPH [6]. The time-scale thermostat, used in the present work, is inspired by the Berendsen thermostat [40], a very common choice in molecular dynamics simulations. The thermal evolution of the mean temperature is given by the solution of the ordinary differential equation

$$
\frac{d \bar{T}}{d t}=\frac{1}{\tau}\left(T_{0}-\bar{T}\right),
$$

where $\bar{T}$ is the arithmetic mean, $\tau$ is a constant that defines the thermalization time scale, and $T_{0}$ is the desired final mean temperature. For the calculation of $\bar{T}$, Eq. (13) is used to assign a temperature to each SPH particle. The solution of Eq. (14) depends on the integration time step $\Delta t$ and is given by

$$
\bar{T}_{\text {new }}=\left(\bar{T}_{\text {old }}-T_{0}\right) e^{-\Delta t / \tau}+T_{0},
$$


where $\Delta t=\max _{i} \Delta t_{i}$ and $\bar{T}_{\text {new }}(t)=\bar{T}_{\text {old }}(t+\Delta t)$. The new temperature $\widetilde{T}_{i}$ of a particle $i$ is then obtained by

$$
\widetilde{T}_{i}=T_{i}\left(\frac{\bar{T}_{\text {new }}}{\bar{T}_{\text {old }}}\right) .
$$

Due to energy conservation in the time integration the particles are only collectively updated according to Eq. (16) only at the maximum size of time steps $\Delta t$.

Note, that for molecular dynamics (MD) the Berendsen thermostat is known to suppress thermal fluctuations, which is true for microscopic simulation methods, but for our mesoscopic scheme, where only the overall mean temperature is scaled by the exponential factor to the desired value, thermal fluctuations are conserved.

If latent heat is released, then $\tau$ defines the half time of the process where the heat is conducted to the heat bath.

\section{Domain size analysis}

The quenched system instantaneously initiates to separate in two phases, the liquid and the vapor phase, respectively. A quantity of interest is how the domain sizes evolves in time, that expresses the extent of decomposition. A common method, that is well established for spinodal decomposition of binary mixtures, is the analysis via the structure factor [1], where the mean domain size can be extracted from its first moment. This is indeed an acceptable method when the density of the phases is roughly the same and the concentration of two liquid species is considered. However, liquid-vapor systems differ in density and, therefore, the growth rates are also expected to differ. Moreover, the mean domain size is not efficient to characterize the dynamics of the system [41], especially for liquid-vapor systems. Therefore, the characteristic length shall be considered separately for each phase [6]. A natural ansatz for the SPH method is to define a threshold density value, usually the critical density $\rho_{c}=2 / 3$, and divide in liquid and vapor particles, $i_{l}$ and $i_{v}$, respectively. The mean of minimum distances to the opposing phase is used to define the characteristic lengths, that belong to the certain phases. Thus, the characteristic length $\xi_{l}$ for the liquid phase reads as

$$
\xi_{l}(t)=\frac{1}{N_{l}} \sum_{i_{l}}^{N_{l}} \min _{i_{v}}\left[\left|\mathbf{r}_{i_{l}}(t)-\mathbf{r}_{i_{v}}(t)\right|\right],
$$

where $N_{l}$ is the total number of liquid particles. Note that the characteristic lengths obtained from this definition leads to smaller values than those from conventional methods. For example, a spherical liquid droplet in a vapor medium had only a length of $1 / 4$ of its diameter. However, the growth rates are not affected by this fact and it is only a linear effect. Furthermore, the analysis results are found to be in excellent agreement with theoretical predictions [6].

\section{Initial conditions}

The particles are initially glasslike distributed. For this purpose, a repulsive body force is applied to randomly distributed particles, which evolve in time until a relaxed state is obtained. Thereupon, the particles are further relaxed by applying the SPH algorithm to the particles at a supercritical temperature, where the system is expected to be stable. The initial configuration is distributed in the described way with $N_{\text {tot }}=10^{5} \mathrm{SPH}$ particles, and $T_{0}=1.5$. The resulting homogeneous initial density $\rho_{0}=0.5$ is used for all simulations. This procedure has the advantage of very small fluctuations with a standard deviation of $\sigma_{\rho}=3.5 \times 10^{-4}$. Periodic boundaries are applied to a cubic box with side lengths $L=1$. Thus, the total mass is $M_{\text {tot }}=\rho_{0} * L^{3}=0.5$ and the particle mass is given by $m_{i}=M_{\text {tot }} / N_{\text {tot }}$. The density specific thermal conductivity is set to $\kappa_{0}=0.05$, which causes the restriction $\kappa(\rho)=\kappa_{0} \rho \in[0,0.1]$, because $\rho \leqslant 2.0$ due to the $\mathrm{vdW}$ theory. In order to reduce numerical errors, it is found that a reasonable maximum integration time step is $\Delta t_{\max }=5 \times 10^{-4}$, evolving up to $t_{\max }=50$, where all runs are eventually in local equilibrium. The number of smoothing neighbors for the kernel interpolation is set to $N=250$.

\section{SIMULATION RESULTS}

The evolution of the early stage of spinodal decomposition is usually described by the rise of fluctuations. So far, the early stage received little attention compared to the late stage of domain growth and coarsening. The time scale of the thermalization shows to be responsible for the type of growth in the early stage. Therefore, it indirectly affects also the late stage. The process of thermalization also influences the evolution of the mass fraction between the phases, where shorter time scales lead to a faster mass allocation to the phases. The density evolution is also affected. A slow thermalization causes the once separated heated phases to evolve along the coexistence curve, as long as the relaxation to the desired temperature is in progress.

The simulations begin right after the instantaneous quench, which is realized by setting both thermostat parameters, namely the initial and the desired temperature $T_{0}$, to a value lower than the critical temperature, such that the system is completely unstable. First, the deep quenches with $T_{0}=0.8$ are presented, where the time scales $\tau$, defined by Eq. (14), are varied by several orders of magnitude between $10^{-4}$ and $10^{1}$. Additionally, a pure thermal (PT) simulation without any thermostat and a simulation with a scaling thermostat (ST) are also performed to facilitate the comparison. Due to the expectation that the dynamics are affected by the actual system temperature, it is further examined by a comparison of several off-critical quenches of varying depths. For this purpose, simulations with the desired temperatures $T_{0}=0.6,0.7,0.8,0.9$, and 1.0 are performed. For each temperature the time scale $\tau$ is varied from $10^{-4}$ to $10^{1}$ by factors of 10 .

In Fig. 1 a comparison of the rendered densities of cross sections from snapshots of the early stage of decomposition for the $T_{0}=0.8$ quench is shown up to $t=3.0$, when the phases are expected to already be separated and the late stage of domain growth has started in all simulations. The snapshots already reveal several features, which will be focused on in more detail later. It becomes apparent that all simulations are very similar in the early evolution, whereas the further evolution can generally be distinguished between two different types. This can mostly be seen by a comparison between the snapshots at $t=1.5$ and 3.0, where the interfaces are still diffuse for the larger time scales $\tau \gtrsim 1$, such as $\tau=10^{1}$ 


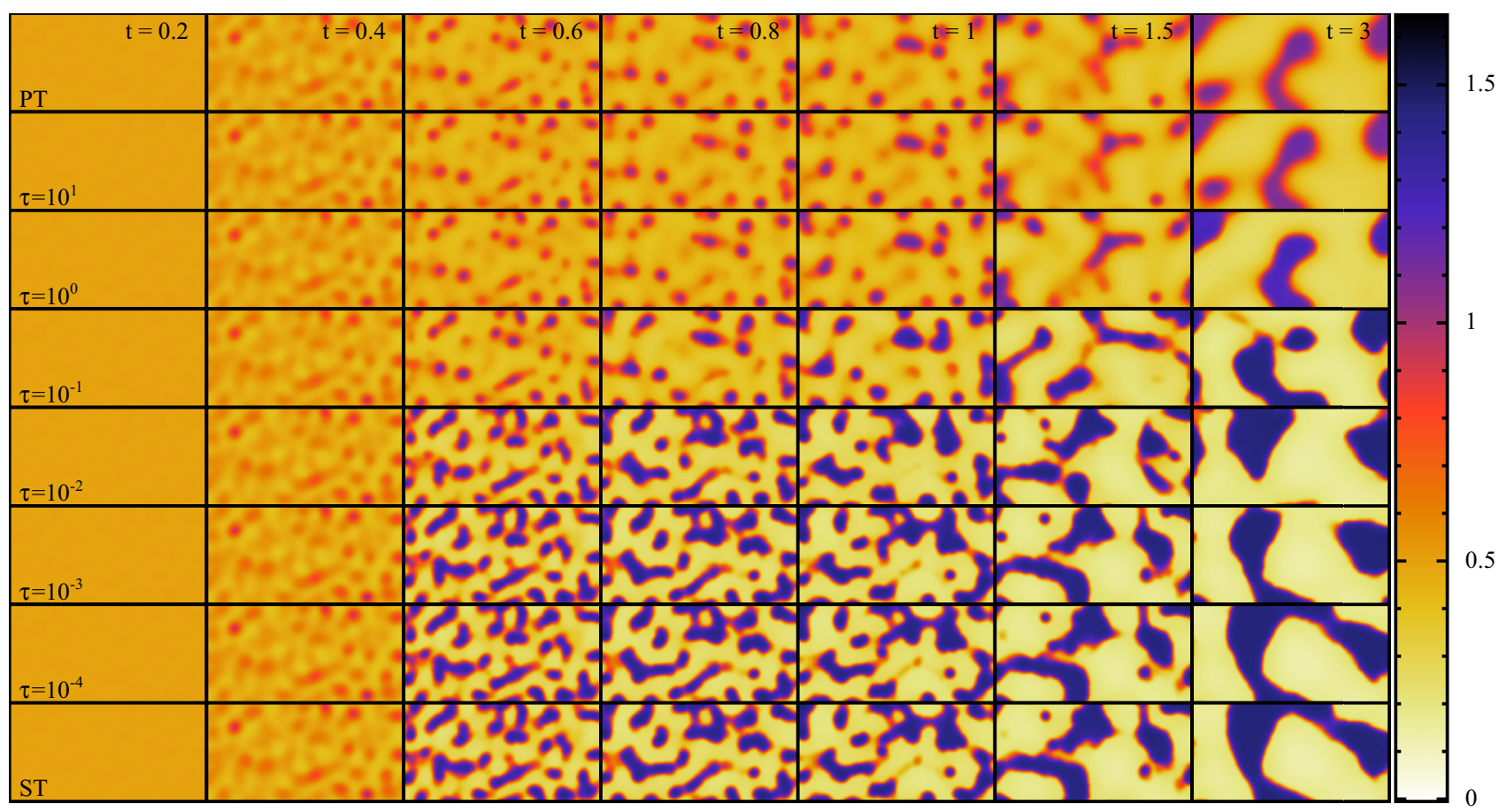

FIG. 1. Density rendered cross section slices (with thickness 0.02 in $z$ direction and only half of the box in $y$ direction is shown) of the early stage at the times $t=0.20,0.4,0.6,0.8,1.0,1.5,3.0$. Exemplarily the simulations with $\tau=10^{1}, 10^{0}, 10^{-1}, 10^{-2}, 10^{-3}, 10^{-4}$ (from top to bottom), compared with the runs of pure thermal evolution (PT) and scaling thermostat (ST), are shown with a side length of size of the box $L=1$. Note that the actual shapes of the $3 \mathrm{D}$ structures can not be determined in this $2 \mathrm{D}$ representation.

and $10^{\circ}$ and the PT simulation. In contrast, sharp interfaces are formed for the shorter time scales $\tau \ll 1$, particularly $\tau=10^{-3}$ and $10^{-4}$ and the ST simulation. A transition point between these contrary images can qualitatively be placed at $\tau \approx 10^{-1}$. This particular run seems to start similar to the $\tau \gtrsim 1$ simulations, but eventually evolves similar to the $\tau \ll 1$ runs. Despite the respective evolution, all simulations have in common that the phases, once separated, undergo a coarsening process driven by surface tension, where the domains initiate to grow. Already at this point, a differentiation between liquid and vapor phase is reasonable, since the respective domains do not coincide in their sizes. A more detailed discussion on the requirement of distinction can be found in Appendix A.

In Fig. 2 the phase diagram for the $T_{0}=0.8$ runs is shown, wherein the median of the density values and the mean temperatures for the respective phase are plotted in steps of $\Delta t=0.05$. The calculation starts with the exceed of the threshold density at $\rho_{c}=2 / 3$, whereby this point in time is 0.35 and unaffected by the actual choice of $\tau$. The use of the median of the density values, instead of the commonly used arithmetic mean, has some advantages. Mainly, it is the robustness to single strong density deviations, which is usually present, when diffuse interfaces are formed [6]. The respective similarities in the evolution between PT and large time scales $\tau \gtrsim 1$, and between ST and short time scales $\tau \ll 1$, are further assured by the evolution of the separated phases in the temperature-density phase diagram. The simulations ST, $\tau=10^{-4}$ and $10^{-3}$, almost perfectly coincide. Also the early evolution of PT and $10^{1}$ evolve roughly equal. So far, it is clear that the late evolution of the $\tau=10^{1}$ run must diverge from PT, because of the time-scale thermostat that decreases the temperature. However, the process is very slow and these simulations do not reach the desired final temperature within the simulated time range. Note that the discrepancy between the end up vapor density values and the theoretically predicted binodal value mainly belongs to the resolution, since the vapor phase has only very few particles. However, it also belongs

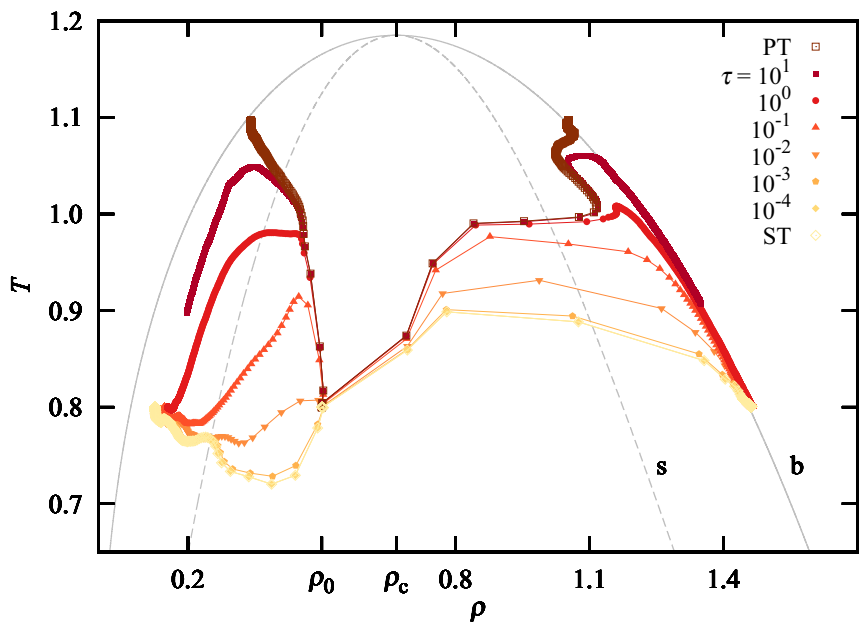

FIG. 2. Evolution in the temperature-density phase diagram for $T_{0}=0.8$. Data sets are connected by lines. The pure thermal PT, several thermostat time scales $\tau$, and the scaling thermostat ST (from top to bottom, in steps of $\Delta t=0.05$ ) are shown. PT and ST are highlighted by open dots. The gray lines represent the binodal (solid) and spinodal (dashed line) curves from vdW theory and Maxwell construction. 
to the choice of mean, because the median is namely very robust, but does not coincide with the peak value of the density distribution.

\section{A. Early stage demixing}

The behaviors of PT and ST are well examined [5,6,42], more precisely, the quasi-isothermal ST behavior, where the vapor and liquid phase are observed to contrarily cool down or heat up, respectively. This phenomenon becomes clear from Eq. (13), where the thermal conductivity is finally responsible for the equilibration of the system. In contrast, in PT simulations both phases, liquid and vapor, are observed to heat up, but with the vapor temperature temporally lagging behind the liquid temperature. It can be explained by conduction, which is responsible for the heating of the vapor by the hotter liquid. This leads to a stagnation of heating of the liquid phase until the vapor is heated up to thermal equilibrium, which is followed by an increase of the overall mean temperature. The results from the PT and ST simulations in this work slightly differ from those obtained in Ref. [6]. This must be addressed to the physically more accurate formulation of the density dependent thermal conductivity $\kappa(\rho)$, given by Eq. (10).

Both the $\tau=10^{1}$ and $10^{0}$ runs show the stagnation of heating, which is actually characteristic for thermal simulations. The coexistence density of the liquid phase that is reached corresponds to a higher temperature than the predicted $T_{0}$. Later in progress, in the simulations with thermostat, the temperature decreases and evolves along the coexistence curve to the desired temperature. Solely, the PT run persists in that heated state.

The demixing process and the growth of the domains can be investigated by means of the characteristic lengths $\xi$, defined in Eq. (17). The lengths are shown in Figs. 3 and 4 for the liquid and vapor phase, respectively. For the reason of better comparability the curves are each shifted by factors of $10^{0.1}$.

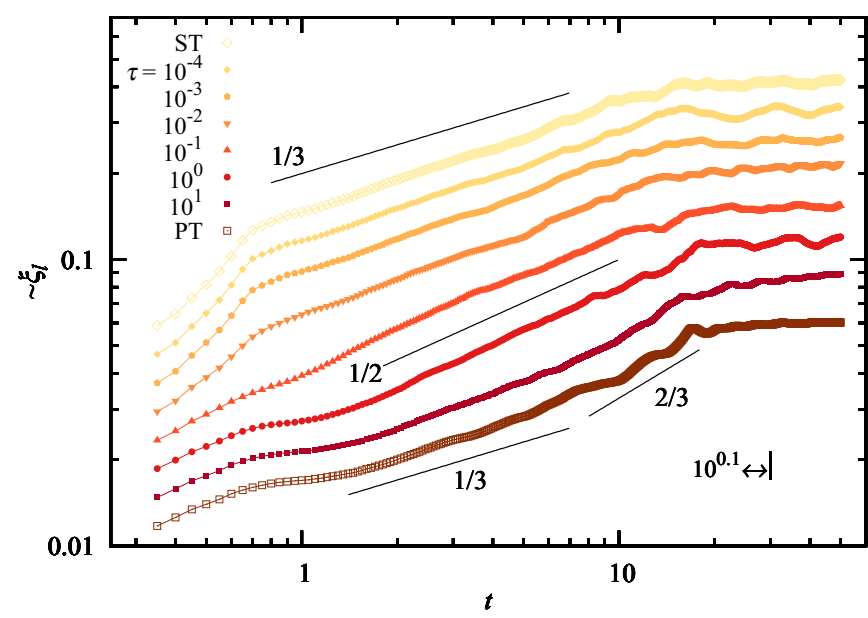

FIG. 3. The characteristic lengths of the liquid phase $\xi_{l}$ for $T_{0}=0.8$, plotted in steps of $\Delta t=0.05$, additionally connected by lines. PT and ST are highlighted by open dots. The data sets are each vertically shifted by factors of $10^{0.1}$ due to a better comparability. The factor is also shown true to scale. The black lines indicate power laws to guide the eyes.

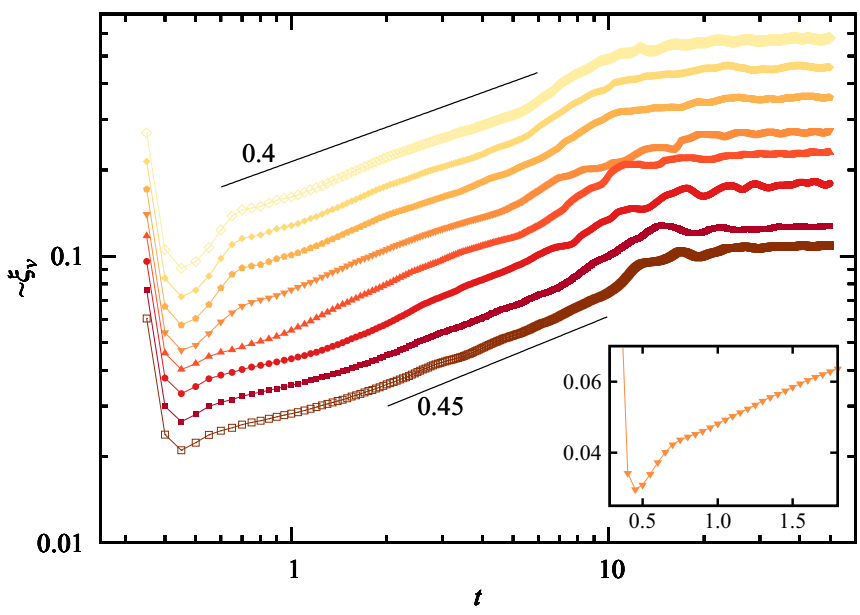

FIG. 4. The characteristic lengths of the vapor phase $\xi_{v}$ for $T_{0}=0.8$, plotted in steps of $\Delta t=0.05$ connected by lines (coding as in Fig. 3). The data sets are each vertically shifted by factors of $10^{0.1}$ due to a better comparability. The black lines are shown to guide the eyes. The inset shows a nonlogarithmic plot of the $\tau=10^{-2}$ curve in the early stage.

It is due to the initial density $\rho_{0}=0.5$ that per definition the one-component fluid is vapor in the initial state. That means in effect $\xi_{v}(0)=1$, followed by a rapid decrease, whereas the liquid phase initiates at $\xi_{l}(0)=0$, which further strongly increases. The initial state also determines the composition that eventually manifests in the separated system [4]. Here, a minor liquid phase in a major vapor phase is expected, which is why the main focus is on the liquid phase, whereas the vapor is only considered as the solvent.

The early stage takes place right after the instantaneous quench, and the very early dynamics are dominated by the rise of so called "homophase fluctuations" [1], which arise from the initial noise of the density field. The point in time of demixing is unaffected by the choice of $\tau$. Therefore, it appears to suggest it also for the rise of fluctuations. However, the further evolution of the $\xi_{l}$ curves in Fig. 3 differ. It can be distinguished between the two beforehand mentioned different procedures. First, for ST and $\tau \ll 1$, the initial growth has rather more exponential growth character, which is followed directly by a power law scaling in the late stage. In contrast, for PT and for $\tau \gtrsim 1$, the initial growth seems to scale with an ordinary power law, which is followed by a plateau, where the growth of $\xi_{l}$ stagnates, also followed by the ordinary power law scaling of the late stage. It is obvious that the type of initial growth, whether it is described by an exponential or an ordinary power law, depends on the choice of $\tau$. The change is, as already qualitatively mentioned, placed at $\tau \approx 10^{-1}$, more precisely, between $\tau=10^{-2}$ and $10^{-1}$.

Since this growth behavior in the early stage is also expected to be strongly affected by the actual temperature of the system, it will now be discussed in more detail by comparison of several quench depths.

The type of growth of the domains in the early stage is found to differ between the simulations. Interfaces become diffuse, unless the interfaces will not be formed before the intermediate stage. These are the simulations with slow thermalization 


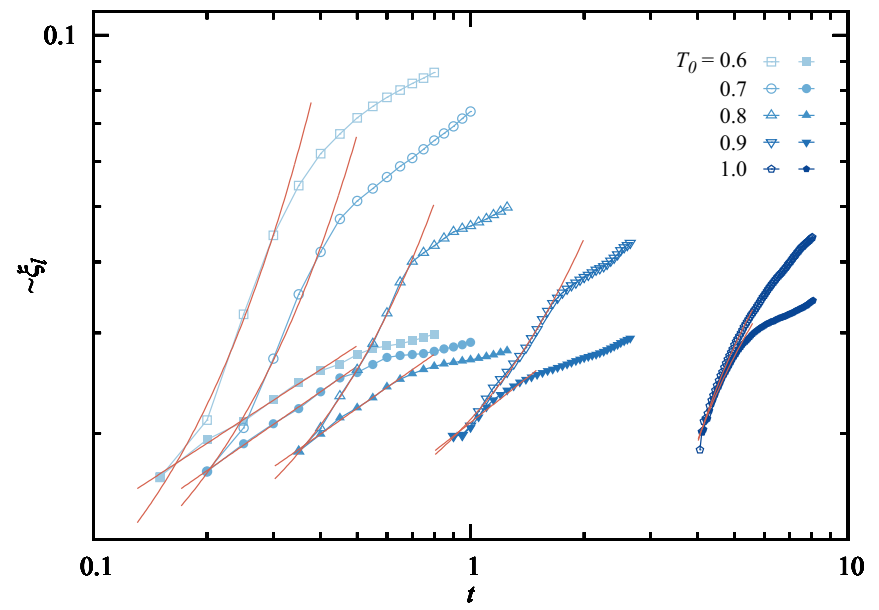

FIG. 5. The early and, if apparent, the intermediate stages of $\xi_{l}$ for $\tau=10^{-4}$ (open symbols) and $10^{1}$ (filled symbols) for different quench depths at $T_{0}=0.6,0.7,0.8,0.9$, and 1.0. Overlaying curves (red) belong to least square fits with the parameters presented in Table I.

$\tau \gtrsim 1.0$, which increase the temperature, or apparently those with the temperature already above the threshold, i.e., $T_{0} \gtrsim$ 1.0. In Fig. 5 a comparison of the early stages of the characteristic lengths $\xi_{l}$ for different quench depths is shown. As discussed in Appendix A, the $\xi_{v}$ are not suitable to realistically capture the growth behavior in the early phase, which is due to the initial rapid decrease of the curves. Therefore, only the lengths of the liquid phases are evaluated here. Representatively chosen for the fast and slow thermalization are the simulations with $\tau=10^{-4}$ and $\tau=10^{1}$, respectively. The $\xi_{l}$ in the very early stage are used for a least square regression. It turns out that the best fitting regression function strongly depends on both the actual depth of the quench and thermalization time scale. The fitting parameters, which belong to the overlaying straight curves in Fig. 5, are listed in Table I. The corresponding functions, that are used for the

TABLE I. The parameters $\beta$ (with asymptotic standard error ${ }^{\mathrm{a}} \delta \beta$ ) are obtained via least square fitting of the $\xi_{l}$ in Fig. 5 within suggested best fit intervals. The expected types of growth are exponential (exp), power law scaling (scale), or logarithmic (log). The appearance of an intermediate stage is synonymous to the plateau in the $\xi$ curve.

\begin{tabular}{cccclc}
\hline \hline$T_{0}$ & $\log _{10}(\tau)$ & Type & Fit interval & $\beta( \pm \delta \beta)$ & Plateau \\
\hline 0.6 & -4 & $\exp$ & $0.15-0.30$ & $6.88(43)$ & no \\
0.7 & -4 & $\exp$ & $0.20-0.40$ & $4.56(25)$ & no \\
0.8 & -4 & $\exp$ & $0.35-0.70$ & $2.23(4)$ & no \\
0.9 & -4 & exp & $0.90-1.60$ & $0.73(1)$ & no \\
0.6 & +1 & scale & $0.15-0.40$ & $0.43(1)$ & yes \\
0.7 & +1 & scale & $0.20-0.45$ & $0.460(8)$ & yes \\
0.8 & +1 & scale & $0.35-0.65$ & $0.46(1)$ & yes \\
0.9 & +1 & scale & $0.90-1.45$ & $0.52(3)$ & yes \\
1.0 & -4 & $\log$ & $4.05-5.55$ & $0.401(3)$ & no \\
1.0 & +1 & $\log$ & $4.10-5.25$ & $0.436(5)$ & yes \\
\hline \hline
\end{tabular}

$\overline{\text { a Asymptotic standard error expresses the accuracy in accordance to }}$ the calculated characteristic lengths.

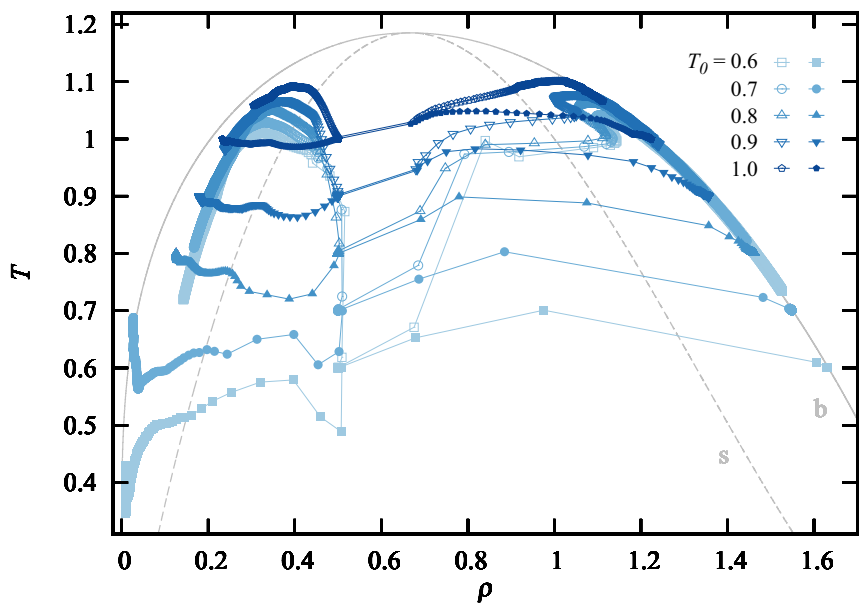

FIG. 6. Phase diagram for several quench depths for the thermalization time scales $\tau=10^{1}$ (open) and $\tau=10^{-4}$ (filled) up to the simulation time $t_{\max }=50$.

regression, are as follows. Three different types of growth can be distinguished. An exponential increase $\xi \sim e^{\beta t}$ is observed for $\tau=10^{-4}$ and deep quenched systems with $T_{0}=0.6,0.7$, 0.8 , and 0.9 . As $\tau$ becomes larger, the growth rates successively alter and follow an ordinary power law scaling, given by $\xi \sim t^{\beta}$. The best fitting values for this range of $\tau$ appear to suggest an exponent $\beta \approx 1 / 2$. For higher $T_{0}$ values, e.g., the $T_{0}=1.0$ run, the growth of fluctuations seems to be unaffected by the choice of $\tau$. Furthermore, the type of growth of the $\xi_{l}$ curves has changed to logarithmic scaling of the form $\xi \sim \log _{10}(\beta t)$, for both, $\tau=10^{-4}$ and $10^{1}$, not least qualitatively, but also supported by the fitted values.

The differing behavior in the early stage can be clarified with a view on the temperature-density plane of the phase diagram, which is shown for all quenches in Fig. 6 for the simulations with $\tau=10^{1}$ and $10^{-4}$. Note that the $\tau \ll 1$ simulations are very similar to the corresponding ST runs, for which, as recently described in Ref. [6], both phases evolve in different directions, forced by the caloric $\mathrm{vdW}$ EOS in Eq. (13). The volume expansion of the vapor phase causes a temperature decrease of the phase, and the liquid temperature increases, caused by the increase of $\rho_{l}$. Eventually, thermal conductivity forces the system to thermal equilibrium. However, the $\tau \gtrsim 1$ simulations show contrary behavior, because the mean temperature of the system is allowed to deviate from $T_{0}$, which is an effect of the slow thermalization. Therefore, the mean temperature is expected to finally evolve in the same direction of that phase that accretes most of the mass. Therefore, it depends on the initial $\rho_{0}$. In our setup, the liquid becomes the mass rich phase, whereas the vapor phase still tends to decrease the temperature. Therefore, the vapor phase gets heated through thermal conduction, which affects the vapor temperature to generally lag behind the liquid phase in relation of the total heating of the system. A stagnation of heating in the liquid phase is observed for all initial temperatures except for the $T_{0}=1.0$ simulations. Note, that the exceptional role of the latter also manifests in the differing type of scaling of $\xi_{l}$, which is logarithmic. It can be explained by the prolongation of the dynamics with higher 
system temperatures $[5,6]$, in the way that thermal conductivity already directly affects the demixing in the very early stage. Moreover, $T_{0}=1.0$ is above this temperature, where the heat stagnation can be observed in the remaining simulations.

The equilibration of the temperature imbalance in the early stage can be expected to be mainly driven by thermal conductivity, which is certainly true for $\tau \ll 1$ and for large $T_{0}$. For the $\tau \gtrsim 1$ range it is found that also convective heat transfer plays a decisive role for spinodal decomposition [8]. Convective heat transfer is also responsible for the $\tau$ dependent reduction of the growth rate in Fig. 5. This can be seen from the liquid mass fraction, which is defined by

$$
\mu_{l}=\frac{M_{l}}{M_{\mathrm{tot}}},
$$

where $M_{l}$ is the total mass of the liquid phase. It is due to our definition of the threshold density $\rho_{c}=2 / 3$, that the liquid mass fraction initializes with $\mu_{l}=0$, which is followed by a strong increase. A comparison of the liquid mass fractions for several quenches is shown in Fig. 7. Again, only the curves for $\tau=10^{-4}$ and $\tau=10^{1}$ are exemplarily shown, for $\tau \ll 1$ and $\tau \gtrsim 1$, respectively.

For the latter, a comparison with the temporal evolution in the phase diagram reveals an unexpected coinciding feature. Mass becomes not only reduced, but a backflow from liquid to vapor is observed in the $\mu$ curves. It takes place at exactly the same time, when the stagnation of heating is observed for the deep quenched simulations $T_{0}=0.6,0.7,0.8$ in the phase diagram. The strength of this convection driven mass flow increases with the depth of the quench. The $\mu_{l}$ of the deep quenches further undergo local minima, followed by a slow increase until equilibrium is reached, whereas for $T_{0}=0.9,1.0$ the backflow diminishes and only a halted increase is observed. The minima are found to take place at exactly the same time, where the liquid density starts to decrease in the phase diagram (see Fig. 6) at the reflection point. Moreover, the further evolution of the $\mu$ curves coincide with the time interval, where the plateau appears in the characteristic lengths.

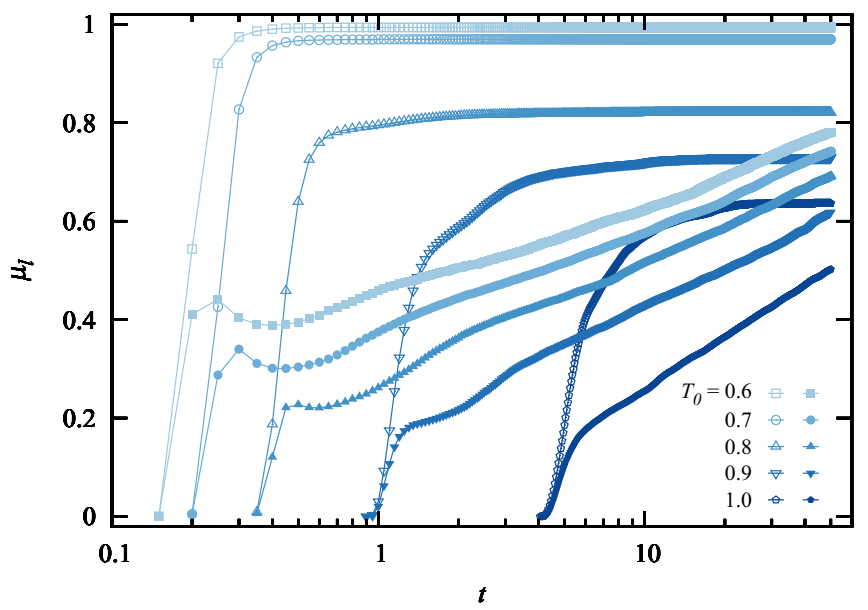

FIG. 7. Liquid mass fractions $\mu_{l}$ for several quench depths over time $t$ for the thermalization time scales $\tau=10^{1}$ (filled symbols) and $\tau=10^{-4}$ (open symbols).
However, for high temperatures the morphological evolution slows down and droplet nucleation dominates spinodal decomposition and the diffuse interfaces are formed simultaneously with the separation of the phases [6]. This leads to the instantaneous formation of spherical shaped droplets. The mass backflow in the evolution of $\mu$ vanishes, e.g., at the $T_{0}=1.0$ curve, as it can be seen in Fig. 7. Additionally, a prolonging behavior of the dynamics is also observed for larger $\tau$, which must be mainly due to the rapid increase in temperature. In contrast, the thermal relaxation, back to the desired temperature $T_{0}$, in these systems is slow. Therefore, the temperature is allowed to exceed a certain threshold value, which is at $T_{\text {th }} \approx 1.0$, as it can be seen in Fig. 6 .

\section{B. Intermediate stage and diffuse interfaces}

The physical origin of the stagnation of growth in the characteristic lengths $\xi_{l}$ in Fig. 3 temporally placed at $t \approx 1$ has been a basis for discussion. Thereby, this growth stagnation is expected to depend on several system parameters, such as surface tension, quench depth, thermal conduction, or intensity of the initial noise $[3,5,16,17,43]$. This growth stagnation will be further on referred to as plateau. However, the appearance and, therewith related, the duration of this plateau in the $\xi_{l}$ curves is strongly connected to the actual choice of $\tau$. It is not observed for $\tau \leqslant 10^{-2}$ and ST. Furthermore, it is associated with the beforehand described change in the type of scaling behavior of the early stage. The plateau becomes observable at $\tau=10^{-1}$ and achieves longer durations for larger $\tau$ values.

An explanation for the plateau can be given by taking a closer look at the phase diagram in Fig. 2. It is placed right after the stagnation of heating at the reversal point of the liquid density. At this point the density is about to decrease and the temperature starts to increase again. For both runs, the $\tau=10^{1}$ and PT, the density reaches a local maximum value at $t=0.7$ and does not further increase until $t \approx 2.0$. The distinctness of the two reflection points in time appear to suggest it as an intermediate stage. During the time interval both phases, the liquid and the vapor, simultaneously decompress, which could plausibly be explained by two reasons. One is that both phases expand their volume. However, this is not possible due to the finite size of the simulation box. The second is that at least one phase excludes mass to form diffuse interfaces. Exemplarily, the density histograms of the $\tau=10^{1}$ and $10^{-4}$ runs for $T_{0}=0.8$ at different times are compared, as shown in Fig. 8. It reveals that the latter explanation is more reasonable. A movement of the peaks in the direction of lower densities is accompanied by the broadening of both the vapor and the liquid density distribution in the $\tau=10^{1}$ data in Fig. 8(a). In the $\tau=10^{-4}$ simulation in Fig. 8(b), the density peaks drift apart and the interface remains sharp even at late times. This can be seen in more detail from the inset box in Fig. 8(b), where a zoom to the vapor phase is shown. The expectation that the phases are excluding mass to form interfaces for large $\tau$ and PT is supported by the inset box in Fig. 8(a), where the zoom to the liquid phase is shown. Along with the process of phase separation, the liquid peak is rising (light-red arrow), and moves in the following to a lower density value (dark-red arrow). Simultaneously, a second peak arises at the 

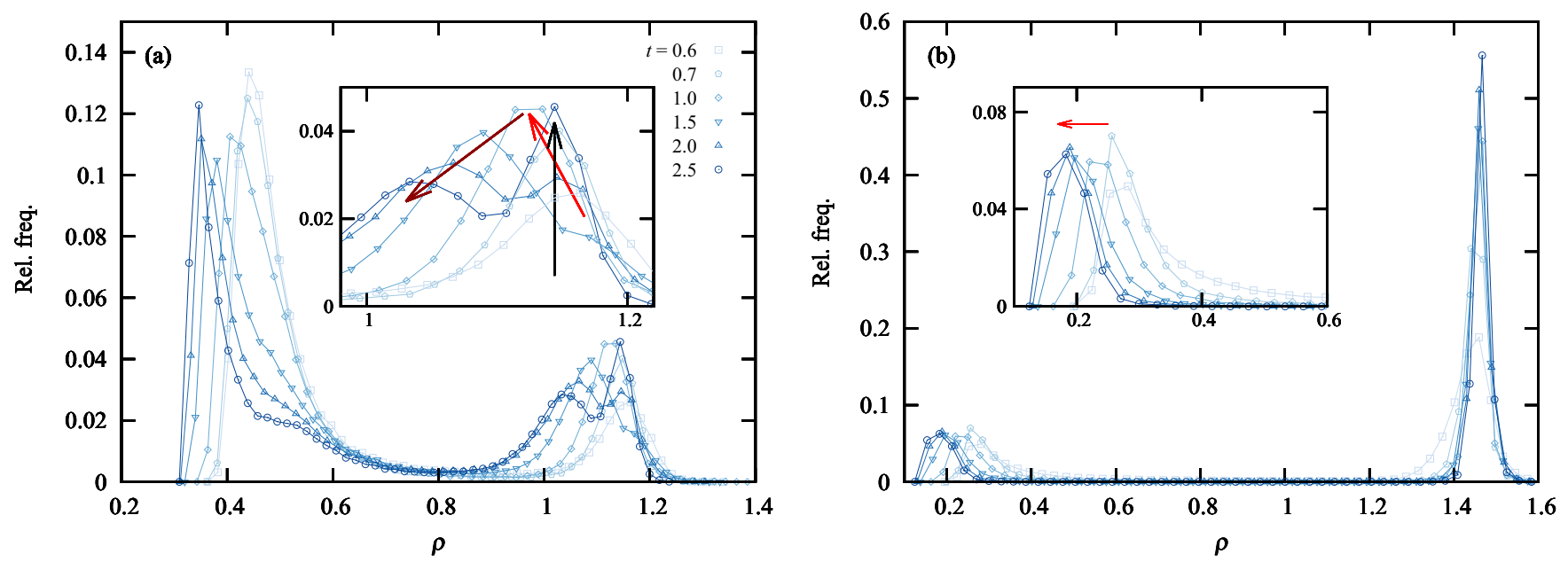

FIG. 8. Density histograms of the simulations (a) $\tau=10^{1}$ and (b) $\tau=10^{-4}$ at different times, using 50 bins. Note that the plot ranges in panels (a) and (b) differ. The insets enlarge detailed regions of the histograms. The arrows mark the peak movements (temporally ordered from light to dark).

corresponding coexistence density (black arrow) as indicated by the arrows.

Therefore, the duration of the plateau in Fig. 3 is not only affected by the rapidity in achieving the coexistence density, but also by forming diffuse interfaces. As it is shown in Fig. 1, diffuse interfaces are only formed in simulations with large $\tau$ and PT, in contrast to the evolution of the remaining simulations. Thus, only for high temperatures $T \gtrsim 1.0$, interfaces become diffuse [6]. This is why the plateau can only be observed for simulations that exceed this temperature threshold. This even applies for simulations with higher initial temperatures but also for large $\tau$ values, since the latter allow for latent heating of the system. The temperature dependency will now be discussed in more detail.

As already mentioned above, the subsequent dynamic growth behavior is directly connected to the heating. It is characterized by two possible scenarios. Due to the actual choice of $\tau$ the late stage can directly follow the early stage. The domain growth in the late stage can then be described by theoretically predicted scaling laws. A detailed discussion can be found in Appendix B. In the second scenario, an intermediate stage occurs, which manifests in the formation of a plateau in the $\xi_{l}$ curves. The intermediate stage is placed right between the early stage, where homophase fluctuations arise, and the late stage, where coarsening and domain growth are the dominant processes. It shall be clarified here how the occurrence and the duration of this intermediate stage is affected by both quench depth and the choice of $\tau$.

It has already been clarified in the previous section that the plateau can only be observed for a certain range of parameters, such as $\tau \gtrsim 1$. This is assured by the $\xi_{l}$ curves in Fig. 5, where the plateau is only apparent for the $\tau=10^{1}$ curves. The appearance of the plateau is correlated to the formation of diffuse interfaces. A further quantification is given with the phase diagram in Fig. 6. The plateau starts exactly at the reflection point $t_{1}$, where the density value of the liquid phase $\rho_{l}$ begins to decrease. The end of the intermediate stage is given by the second reflection point $t_{2}$, where $\rho_{l}$ initiates to reincrease. The corresponding time interval, defined by the two reflection points, gives a duration $\Delta t_{\tau, T_{0}}$, which depends on the actual choice of $\tau$ and $T_{0}$. The values for those simulations, where the intermediate stage is apparent, are summarized in Table II. They are additionally visualized in Fig. 9, where the relevant region in the phase diagram is shown. It can be seen that this phenomenon only occurs in the metastable region, between the spinodal and binodal curves. Moreover, it is an effect of slow thermalization, where the largest $\Delta t_{\tau, T_{0}}$ are found for the PT runs and become smaller with decreasing $\tau$. Note that the first inflection points $t_{1}$, obtained via the phase diagram, are all consistent with the end times of the fit intervals of the early stage scaling analysis, given in Table I. The PT and $\tau=10^{1}$ have identical $t_{1}$ values. The intermediate stage becomes smaller with shorter thermalization time scales and eventually vanishes at $\tau=10^{-1}$. That shows that the intermediate stage is a phenomenon of systems of weak heat bath coupling.

TABLE II. The table gives the time interval $\Delta t_{\tau, T_{0}}$, that corresponds to the two reflection points $t_{1}$ and $t_{2}$ in the evolution of the liquid phase. Listed are only the simulations where the intermediate stage exists.

\begin{tabular}{lcccc}
\hline \hline$T_{0}$ & $\log _{10}(\tau)$ & $t_{1}$ & $t_{2}$ & $\Delta t_{\tau, T_{0}}$ \\
\hline 0.6 & PT & 0.50 & 2.35 & 1.85 \\
& +1 & 0.50 & 1.75 & 1.25 \\
0.7 & PT & 0.55 & 2.35 & 1.80 \\
& +1 & 0.55 & 2.10 & 1.55 \\
& +0 & 0.7 & 0.90 & 0.20 \\
0.8 & PT & 0.65 & 2.75 & 2.10 \\
& +1 & 0.65 & 2.65 & 2.00 \\
& +0 & 0.90 & 1.35 & 0.45 \\
0.9 & PT & 1.55 & 4.25 & 2.70 \\
& +1 & 1.55 & 3.40 & 1.85 \\
& +0 & 2.00 & 2.50 & 0.50 \\
1.0 & PT & 6.20 & 9.70 & 3.50 \\
& +1 & 6.25 & 7.15 & 0.90 \\
\hline \hline
\end{tabular}




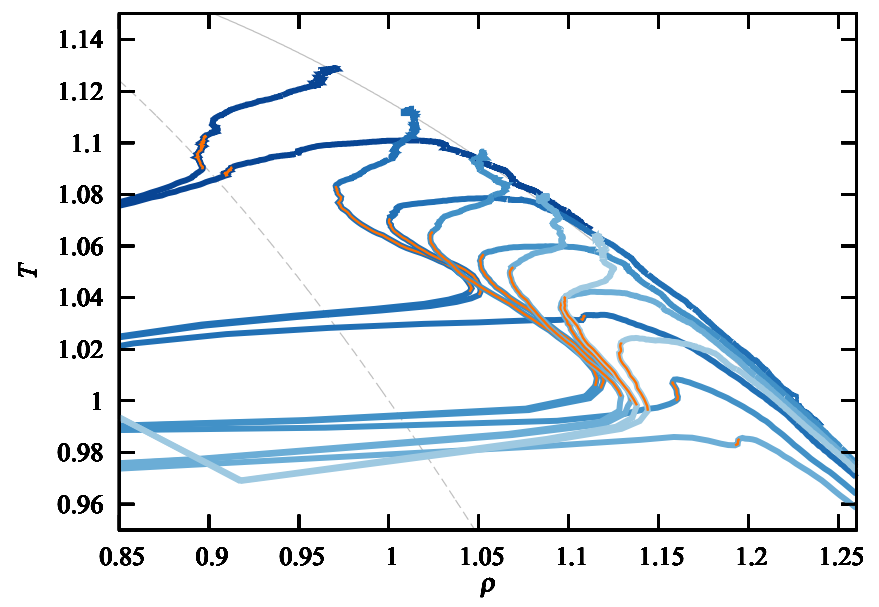

FIG. 9. Evolution in the phase diagram for several quench depths and thermalization time scales, where the intermediate stage is apparent. Color coding as in Fig. 6. The time intervals $\Delta t_{\tau, T_{0}}$ are highlighted.

\section{CONCLUSION}

Our investigation mainly focuses on the dynamics in the early and intermediate stages of spinodal decomposition. The late stage is expected to only slightly influence the actual separation process, since it is dominated by the growth of domains of the already separated phases. Moreover, the late stage is probably the already best investigated of these three stages. The therein involved physical processes are well understood and can be described by scaling laws for the growth of the characteristic lengths. However, the dynamics through the meta- and unstable regions, which lie below the binodal and spinodal curves, respectively, are placed in the early and intermediate stages of the separation process. There is a lack of knowledge concerning the thermal evolution and its effect on the dynamic evolution in that stage. Moreover, the respective temperature evolution is also influenced by the strength of coupling to an external heat bath.

The SPH method, which is herein used for the simulations, can give deeper insights to the thermal evolution of the early phase. Moreover, it is easy to follow the evolution of the separated phases in the phase diagram, and characteristic lengths can easily be calculated by the use of the SPH method. Besides, the computational effort is very modest, as a typical single simulation with $10^{5}$ particles on 48 cores only takes about $15 \mathrm{~h}$.

An exponential decaying thermostat is introduced that allows for the predefinition of a thermalization time scale, on which the heated system relaxes to a desired temperature. The comparison to an instantaneous scaling thermostat shows that the respective choice of the thermostat has a serious effect on the resulting dynamics of the phase separation process. Moreover, the scaling thermostat can be understood as a system with very fast thermal relaxation, as well as in the opposing limit a very slow relaxing system can be understood as a pure thermal simulation. This is shown by a successive variation of the time scale. Note that the matches with these limits are observed to be independent from the actual temperature of the system. The final equilibrated states are in excellent agreement with vdW theory and unaffected by the strength of coupling. The meaningfulness of varying the thermalization time scale is clarified by a closer view on these limits. Isothermal simulations are close to theoretical approaches, which have mostly only phenomenological nature and are, therefore, hard to put into practice. In contrast, a pure thermal evolution is experimentally unrealizable, since already the quench to the spinodal region must be performed. Therefore, it is more realistic to consider systems in between, which allow for both latent heating and thermal relaxation at a certain time scale.

In contrast to the common analysis methods, the requirement for a separated analysis of the liquid and vapor phase is stated. A comparison of the evolution of fast thermalization systems in the phase diagram shows that the two phases evolve contrarily in both density and temperature.

In the early stage, the type of growth of the characteristic lengths differs by the actual choice of thermalization time scale. Short time scales show a fast exponential increase in the characteristic length, whereas at long time scales the lengths grow slower, which is described by an ordinary $1 / 2$ power law. The slower growth rate arises from a mass transfer between the phases which is directed contrary to the expected demixing behavior. The mass flow, which is directed backwards from the arising liquid phase to the vapor phase, is interpreted as thermal convection. In systems with high temperatures the type of growth is independent of the time scale, where the fit calculations suggest a logarithmic scaling behavior.

In slow thermalization systems, the system temperature initially increases due to latent heat followed by a stagnation of that increase in temperature in the liquid phase, while the density further increases. This is not observed at short time scales. By comparison, the nearly identical evolution of pure thermal simulations shows that the stagnation of heating is no effect of the thermostat. It is rather driven by thermal conduction, where the colder vapor phase tends to retard the increase in temperature. Additionally, an intermediate stage appears in systems with slow thermalization, where the growth of the characteristic lengths is reduced and forms a plateau. This halted growth is explained by the formation of diffuse interfaces between the separated phases. They only form at high temperatures, which explains that the plateau appears either at systems that already reached high temperatures or at systems that heat up.

The thermalization thermostat excellently closes the gap between the pure thermal and scaled temperature simulations. This is not only assured by a comparison of the snapshots, but also by the evolution in the phase diagram and by the comparison of the characteristic lengths. At short time scales the results are nearly identical to the scaling thermostat even up to the late stage. The large time scales converge to the pure thermal simulation only in the early and intermediate stages. The late stage must differ, which is due to the absence of a thermal relaxation process in PT simulations. However, it is clear that simulations with even larger time scales will also excellently match with the late stage.

For a realistic dynamic behavior, the time scale must be chosen carefully in between the range of the two limits. It is shown that the strength of coupling has a serious impact on the dynamics while the system is in a completely unstable or, at least, metastable state. Moreover, this argumentation is also 
shown to be supported by the qualitative comparison with both experimental and numerical results of other studies. Thus, the SPH method equipped with the time-scale thermostat provides a powerful instrument for the simulational modeling of phase separation dynamics.

\section{ACKNOWLEDGMENTS}

The authors gratefully acknowledge the computing time granted by the John von Neumann Institute for Computing (NIC) and provided on the supercomputers JUROPA and JURECA at Jülich Supercomputing Centre (JSC). The density plots are rendered with SPLASH [45].

\section{APPENDIX A: DISTINCTION BETWEEN LIQUID AND VAPOR}

It is not only the initial density, but also the involvement of temperature and, therewith associated, the strength of coupling to the heat bath that are observed to affect the scaling of the domains. The direct comparison between $\xi_{v}$ and $\xi_{l}$ of the same $\tau$ value further emphasizes the requirement for a distinction between the two phases, e.g., the different power law scalings in the late stage or the differing final equilibrium lengths, where the $\xi_{v}$ is generally larger than the belonging $\xi_{l}$. The latter fact is more distinctive in the curves for $\tau \gtrsim 1$ and PT. The differences in the curves is also mainly due to the asymmetry of the quench and the resulting density distribution.

Note that the plateau can also be observed in the $\xi_{v}$ curves of the weakly coupled systems, plotted in in Fig. 4. It is due to the assumption that both phases are involved to interface formation, as it can be seen in Fig. 8(a). The characteristics of the vapor $\xi_{v}$ curves are generally very similar to that of $\xi_{l}$, but less distinctive than the characteristics of the $\xi_{l}$ curves. Due to the rapid decrease of the $\xi_{v}$ in the early stage, the type of scaling of initial rise of fluctuations can hardly be worked out from the vapor phase. Moreover, for the large $\tau$ and PT simulations, the plateau, where the interfaces are formed, is less distinctive in the $\xi_{v}$ than it is in the $\xi_{l}$ curves.

Nevertheless, it must be noted that the characteristic lengths are in excellent qualitative agreement with observations from experiments of spinodal decomposition of binary mixtures by Poesio et al., where the effect of the quenching rate on the demixing process is investigated [7]. The inset in Fig. 4 exemplarily shows a linearly scaled part from the early stage of demixing of the $\tau=10^{-2}$ curve, which can be qualitatively compared. Even the initial rapid decrease of the curve and a plateaulike stagnation in growth, which is due to their evaluation method via structure factor, can be confirmed by our data. Poesio et al. postulate a linear growth law for the late stage regime, which indeed might be suggested for the small time range, where the growth process is observed. Our simulation method allows us to evolve the system much longer in time, which allows us for a better estimation of the late stage scaling behavior.

\section{APPENDIX B: LATE STAGE DOMAIN GROWTH}

Theoretical predictions for late stage domain growth rates are mainly given for symmetric quenches in binary mixtures and have recently been shown by simulations to deviate in asymmetric quenches [44]. Moreover, the similarity between binary mixture and liquid vapor is only valid for such symmetric quenches. However, a symmetric mass distribution can hardly be preset in liquid-vapor systems, which is contrary to binary mixtures, where a critical quench predetermines the end up composition. Therefore, the resulting growth rates differ from theoretical expectations. Also, the yielded growth exponents strongly depend on the size of the respective choice of fit intervals [4]. For these reasons, it must be emphasized that we do not claim the observation of new growth exponents, but rather use the existing predictions for our interpretation.

The late stage, where the phases are eventually fully separated, is dominated by the coarsening and the coalescence of droplets. It is expected that the time evolution of the characteristic domain size $\xi$ can be described by a scaling law $\xi \sim t^{\alpha}$. The value of $\alpha$ gives rise to the actual physical process, that is responsible for the respective growth behavior. The driving processes are namely the diffusive LS regime ( $\alpha=1 / 3)$, the $\mathrm{VH}(\alpha=1)$, and the IH regime ( $\alpha=2 / 3$ ) [22]. Note that our simulations have no physical viscosity included, hence, the $\mathrm{VH}$ regime is none of the expected regimes here. Thus, it is only expected to observe exponents localized between $1 / 3$ and $2 / 3$. As it was already mentioned, we do not claim new exponents. Therefore, only slope expectations are shown in Figs. 3 and 4 to guide the eyes. However, for large $\tau$ and PT, for which the mean temperatures significantly increase, the physical processes that are responsible for the scaling regimes, are expected to overlap. They give rise to a variation of the exponents, because the $\mathrm{IH}$ regime becomes relevant earlier at higher temperatures [6]. Furthermore, it has recently been shown that growth rates are strongly connected to the actual temperature of the system [5,6]. In comparison with our earlier works the exponents from this work are in very good qualitative agreement. Both the type of growth in the early stage and the formation of the plateau must be considered to affect the late stage scaling behavior.

The respective choice of $\tau$ affects the final mass distribution between the phases. For $\tau \ll 1$, the mass separates instantaneously and also the $\rho_{l}$ and $\rho_{v}$ reach their final coexistence values. In the case of $\tau \gtrsim 1$, the demixing process lasts much longer, so that the masses do not reach the final fraction at the end of the simulations. This prolonged demixing behavior is also in qualitative agreement with the results of other numerical simulations [5]. Moreover, mass transfer is apparent even in the late stage, indicating that the growth processes in the late stage are overlayed by early stage processes. This is one more reason for the difficulty in using theoretical predictions for the late stage, when thermal processes are taken into account. Note that the very final mass fraction in the totally equilibrated state is hard to predict from the beginning. It is not only predefined by $\rho_{0}$, but also by the $T_{0}$ and $\tau$. Since thermal conduction is present in both the simulations with small and large $\tau$, the thermal balance for the latter must be rather driven by convection than by conduction. Furthermore, the characteristic mass transfer, which is given by $\mu_{l}$, is not observed in the $\tau \ll 1$ simulations. Thus, convection only appears if the latent heat from the phase separation process is not led away fast enough. 
[1] K. Binder, Rep. Prog. Phys. 50, 783 (1987).

[2] D. Frenkel and B. Smit, Understanding Molecular Simulation: From Algorithms to Applications (Academic Press, San Diego, 2001), Vol. 1.

[3] A. G. Lamorgese and R. Mauri, Phys. Fluids 21, 044107 (2009).

[4] A. Chaudhri, J. B. Bell, A. L. Garcia, and A. Donev, Phys. Rev. E 90, 033014 (2014).

[5] Y. Gan, A. Xu, G. Zhang, Y. Li, and H. Li, Phys. Rev. E 84, 046715 (2011).

[6] M. Pütz and P. Nielaba, Phys. Rev. E 91, 032303 (2015).

[7] P. Poesio, G. Cominardi, A. M. Lezzi, R. Mauri, and G. P. Beretta, Phys. Rev. E 74, 011507 (2006).

[8] P. Poesio, A. M. Lezzi, and G. P. Beretta, Phys. Rev. E 75, 066306 (2007).

[9] J. W. Cahn and J. E. Hilliard, J. Chem. Phys. 28, 258 (1958).

[10] J. van der Waals, J. Stat. Phys. 20, 200 (1979).

[11] J. W. Cahn, Acta Metall. 9, 795 (1961).

[12] A. Onuki, Phase Transition Dynamics (Cambridge University Press, Cambridge, England, 2002), Cambridge Books Online.

[13] P. Español, J. Chem. Phys. 115, 5392 (2001).

[14] A. Onuki, Phys. Rev. E 75, 036304 (2007).

[15] T. M. Rogers, K. R. Elder, and R. C. Desai, Phys. Rev. B 37, 9638 (1988).

[16] N. Vladimirova, A. Malagoli, and R. Mauri, Phys. Rev. E 58, 7691 (1998).

[17] Y. Gan, A. Xu, G. Zhang, and S. Succi, Soft Matter 11, 5336 (2015).

[18] E. D. Siggia, Phys. Rev. A 20, 595 (1979).

[19] H. Furukawa, Adv. Phys. 34, 703 (1985).

[20] H. Furukawa, Physica A 204, 237 (1994).

[21] I. M. Lifshitz and V. V. Slyozov, J. Phys. Chem. Solids 19, 35 (1961).

[22] A. J. Bray, Adv. Phys. 43, 357 (1994).
[23] W. R. Osborn, E. Orlandini, M. R. Swift, J. M. Yeomans, and J. R. Banavar, Phys. Rev. Lett. 75, 4031 (1995).

[24] V. Springel, Mon. Not. R. Astron. Soc. 364, 1105 (2005).

[25] V. Springel, Annu. Rev. Astron. Astrophys. 48, 391 (2010).

[26] D. J. Price, J. Comput. Phys. 231, 759 (2012).

[27] J. J. Monaghan, Annu. Rev. Fluid Mech. 44, 323 (2012).

[28] H. Wendland, Adv. Comput. Math. 4, 389 (1995).

[29] W. Dehnen and H. Aly, Mon. Not. R. Astron. Soc. 425, 1068 (2012).

[30] S. Rosswog, Mon. Not. R. Astron. Soc. 448, 3628 (2015).

[31] M. Jubelgas, V. Springel, and K. Dolag, Mon. Not. R. Astron. Soc. 351, 423 (2004).

[32] P. W. Cleary and J. J. Monaghan, J. Comput. Phys. 148, 227 (1999).

[33] D. Molin and R. Mauri, Chem. Eng. Sci. 63, 2402 (2008).

[34] S. Nugent and H. A. Posch, Phys. Rev. E 62, 4968 (2000).

[35] Y. Melean and L. D. G. Sigalotti, Int. J. Heat Mass Transf. 48, 4041 (2005).

[36] H. López and L. D. G. Sigalotti, Phys. Rev. E 73, 051201 (2006).

[37] Z. Xu, P. Meakin, and A. M. Tartakovsky, Phys. Rev. E 79, 036702 (2009).

[38] L. D. G. Sigalotti, J. Troconis, E. Sira, F. Peña-Polo, and J. Klapp, Phys. Rev. E 90, 013021 (2014).

[39] O. Kum and W. Hoover, J. Stat. Phys. 76, 1075 (1994).

[40] H. J. C. Berendsen, J. P. M. Postma, W. F. van Gunsteren, A. DiNola, and J. R. Haak, J. Chem. Phys. 81, 3684 (1984).

[41] K. R. Mecke and V. Sofonea, Phys. Rev. E 56, R3761 (1997).

[42] Y. Gan, A. Xu, G. Zhang, P. Zhang, and Y. Li, Europhys. Lett. 97, 44002 (2012).

[43] E. S. Kikkinides and P. A. Monson, J. Chem. Phys. 142, 094706 (2015).

[44] C. Datt, S. P. Thampi, and R. Govindarajan, Phys. Rev. E 91, 010101 (2015).

[45] D. J. Price, Pub. Astron. Soc. Aust. 24, 159 (2007). 